\title{
Predictive Relations between Pre-discharge APIB Scores and Postterm General Movement (GM) Assesment in Very Low Birth Weight Infants
}

\author{
Fujimoto T', Ogaki A', Inoue $T^{1}$, Kawai T', Tanaka M', \\ Kugo $\mathrm{M}^{2}$, Browne $\mathrm{J}^{3}$ \\ 'Rehabilitation Department, Japanese Red Cross Society Himeji Hospital, \\ Himeji, Hyogo, Japan \\ 2 Pediatrics, Japanese Red Cross Society Himeji Hospital, Himeji, Hyogo, Japan \\ ${ }^{3}$ Pediatrics and Psychiatry, University of Colorado School of Medicine, \\ Aurora, Colorado, USA
}

\section{Background}

Non-invasive assessments of at-risk infants are important tools for recognizing individual needs for support and intervention, as well as the prediction of such needs. The APIB (Assessment of Preterm Infant's Behavior) is a sensitive technique for assessing prematurely born infants at early ages ${ }^{1}$ and, similarly, the GMs (General Movements) assessment at two - four months corrected age is used to predict developmental disorder. ${ }^{2}$ APIB is based on the Synactive Theory and the NBAS (Neonatal Behavioral Assessment Scale). ${ }^{3}$ It is a neurobehavioral battery that yields a panel of multicomponent "packages" reflecting the status of various physiological and regulatory systems in preterm infants. The GM's greatest predictive value is around three months postterm (in the "fidgety" GM stage). The GM evaluation of Hadders-Algra yields a Gestalt evaluation of movement complexity and variation, ranking infants as manifesting normal-optimal GMs (NO), normal-suboptimal GMs (NS), mildly abnormal GMs (MA), or definitely abnormal GMs (DA).

\section{Aims}

The aim of this study was to test whether there are systematic relations between APIB system scores before discharge and those of the GMs assessment around three months corrected age. If there are reliable associations between the two measuring tools, the extra predictive powers may prove valuable to early detection of individual needs and to help guide intervention, remediation, and enhance developmental care.

\section{Subjects and Method}

Among the very low birth weight (VLBW) infants admitted to our NICU/GCU from June 2019 to January 2021, 24 cases (10 boys, 14 girls, average birth weeks $29.7 \pm 3.2$ weeks, average birth weight $1143.3 \pm 248.6 \mathrm{~g}$ ) were selected. They were the cases in which APIB could be performed before discharge, and GM assessment performed around three months corrected age. Excluded from the sample were infants with neurological abnormalities such as hypoxic-ischemic encephalopathy (HIE) and periventricular leukomalacia (PVL). We used the mode of the post package status score of the APIB system scores and summary score derived before discharge (mean
$39.3 \pm 1.4$ weeks). We conducted the GM assessments around three months corrected age (average $3.1 \pm 0.6$ months); the classification of Hadders-Algra was used to evaluate in stages with $\mathrm{NO}$ as $4, \mathrm{NS}$ as $3, \mathrm{MA}$ as 2 , and DA as 1 . Then, we examined quantitatively the relations between APIB's post package status score and summary score, and GM assessments. The APIB procedure and scoring was performed by an APIB Professional; the GM assessments were conducted by three physiotherapists with GM training. Statistical examination was carried out using Spearman's rank correlation coefficient (rs), and the risk factor $\mathrm{p}<0.05$ was statistically significant.

\section{Results}

There were statistically significant correlations between the APIB's physiology and regulatory systems post package status scores and GM assessments (physiology system: $r s=-0.46, \mathrm{p}$ $<0.05$; regulatory system: $\mathrm{rs}=-0.44, \mathrm{p}<0.05)$. In contrast to these findings, no significant relations were found with the scores of motor system, state system, attention/interaction system, and examiner facilitation. Also significant was the relation of the APIB summary score and the GMs assessment ( $r s=0.47, \mathrm{p}<0.05)$.

\section{Conclusions}

We found that APIB scores of the physiology and regulatory systems status in VLBW infants in the NICU/GCU predicted their General Movements GM scores at about three months corrected age, during the so-called fidgety period. Not only does the finding suggest the possibility of enhanced prognosis of developmental disorder, but it helps point to specific developmental systems that can be targeted for intervention. Furthermore, these findings also suggest that the physiology system and regulatory system in the neonatal period may affect the subsequent development. Nevertheless, the GMs assessment may be influenced by state. ${ }^{2}$ Thus, we may examine whether stable autonomic function, high self-regulation, and good wakefulness may help explain the pattern of the results. In the future, measures of variability in different infant populations could help clarify such interpretations. Regardless, the present findings elevate the efficacy of a synactive-based method to link to another diagnostic tool for increased predictive power of the need for developmental support.

References

1. Als H, Butler S, Kosta S, \& McAnulty G. The assessment of preterm infants' behavior (APIB): Furthering the understanding and measurement of neurodevelopmental competence in Preterm and Full-Term Infants. Ment Retard Dev Disabil Res Rev. 2005; 11(1): 94-102.

2. Hadders-Algra M. General movements: a window for early identification of early identification of children at high risk for developmental disorders. J Pediatr; 2004;145:S12-S18

3. Brazelton TB and Nugent JK. 2011. The Neonatal Behavioral Assessment Scale. Mac Keith Press, Cambridge. 